\title{
Spectral and Microbial Studies of Some Newly synthesized Schiff Base Derivatives of 2-(1H- benzo[d]oxazole-2-ylthio)- $\mathrm{N}$-(4- acetylphenyl)acetamide
}

\author{
Jayesh Maru*, G. R. Patel, Rakesh Yadav \\ Department of Chemistry, Sheth M. N. Science College, NGES campus, Patan - 384265, India \\ *E-mail address: jay1maru@yahoo.in
}

\begin{abstract}
The author has synthesized novel biological active compounds by condensation of N-(4-Acetylphenyl)-2-(benzooxazol-2-ylsulfanyl)-acetamide with defferent substituted of acid hydrazide in the presence of catalytic amount of acetic acid. A series of benzoxazole having azomethine group were confirmed by various spectroscopic techniques. The new compounds were examined for antibacterial effects again different strain of bacteria and antifungal were high to lowest Minimum Inhibition Concentration (MIC) values.
\end{abstract}

Keywords: Benzoxazole; Acid hydrazied; Azomethine

\section{INTRODUCTION}

Development of antimicrobial agents for the chemotherapy of fungal and bacterial infections represents one of the most significant achievements of this decade in the field of drug chemistry. The rising emergence of acquired resistance to existing antimicrobials to fungal and bacterial infections leads us to synthesize newer antimicrobial agents. Benzoxazole nucleolus is many clinically useful chemotherapeutic agents [1] shown by literature survey. Flunoxaprofen is one of the most standerd drug of benzoxazole moity which is act as an antiinflammatory [2] drug.

There are many drugs which are useful in different diseases containing benzoxazole nucleus. Benzoxazole exhibited a wide variety of interesting biological activity such as antimicrobial [3], antibacterial [4], analgesics [5], antitumor [6], anti-inflammatory [7], antifungal [8], antioxidant [9], etc. Benzoxazole also used as cyclo-oxygenase [10] inhibitors shown by literature survey.

Azomethine are products of condensation of simple or substituted acid hydrazide with simple or substituted Aceto group containing compound in the presence of catalytic amount of acetic acid in the appropriate solvent as a reaction medium and suitable condition. Azomethine constitute an important group of natural products and some of them show a wide range of biological properties such as Antifungal [11], anti-inflammatory [12], anti-bacterial [13], antioxidant [14] etc. 


\section{EXPERIMENTAL}

All the required chemicals and solvents used for the synthesis were purchased from HIMEDIA, LOBA chemie, SDfine chemicals and/or Merck Ltd. Melting point was determined by the open end capillary method and are reported uncorrected at the primary stage. Completion of reaction was monitored by aluminum coated TLC plates (TLC silica gel $60 \mathrm{~F}_{245}$, E. Merck) using different solvent ratio for different steps as mobile phase and spot checked under ultraviolet (UV) light. Bruker Spectrophotometer-400 MHz where Trimethylsilane(TMS) and Dimethyl sulfoxide(DMSO)-d6 was used as solvent for the ${ }^{1} \mathrm{H}$ NMR and ${ }^{13} \mathrm{C}$ NMR. Shimadzu mass Spectrophotometer used for the Mass spectral analysis. Bruker FT-IR alpha-t (ATR) used for the IR spectral data. Perkin-Elmer 2400 CHN Analyzer used for the elemental analysis $(\% \mathrm{C}, \mathrm{H}, \mathrm{N})$.<smiles>CC(=O)c1ccc(N)cc1</smiles><smiles>CC(=NNC(=O)c1ccc(Br)cc1)c1ccc(NC(=O)CSc2nc3ccccc3o2)cc1</smiles>

Scheme 1. Synthetic route for the preparation of title compounds $5 \mathrm{a}-1$.

\subsection{Synthesis of N-(4-acetylphenyl)-2-chloroacetamide (Comp.2)}

The Synthesis of N-(4-acetylphenyl)-2-chloroacetamide (Compound 2) was carried out by reacting 4 -amino acetophenone $(0.01 \mathrm{~mol}, 135 \mathrm{gm} / \mathrm{mol}, 1.35 \mathrm{gm})$ with chloroacetylchloride $(0.015 \mathrm{~mol}, 113 \mathrm{gm} / \mathrm{mol}, 1.19 \mathrm{ml})$ and Triethylamine $(4-5$ drops $)$ using DMF $(30 \mathrm{ml})$ as 
solvent. The reaction mixture was stirred for 4 hours used the megnatic stirrer at Room temperature. The completion of reaction was monitored by TLC with Mobile phase Toluene:Acetone $(7: 3)$. The solution was poured into ice water $(50 \mathrm{ml})$. The product obtained was filter, dried and crystalline in Ethanol.

Compound 2 : Solide browne crystals; Yield 85\%; M.P. $154 \mathrm{C}^{\circ}$; IR ( $v_{\max }, \mathrm{cm}^{-1}$, ATR): 740 (C-Cl str.), 1413 (C=C str. Aromatic ring), 1640 ( $\mathrm{C}=\mathrm{O}$ str.), $2750\left(\mathrm{CH}_{2}\right.$ str. methylene),

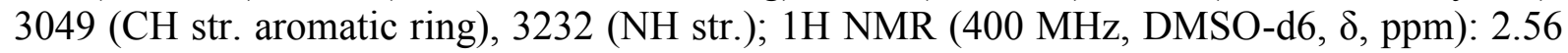
(3H, s, -CH3), 4.89 (2H, s, -CH2), 7.70-8.10 (4H, d, Ar-H), 9.97 (1H, s, -NH); 13C NMR

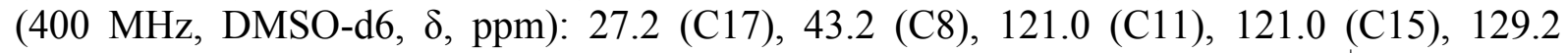
(C12), 129.2 (C14), 137.2 (C13), 166.0 (C9), 198.2 (C16); (MS (m/z): $212\left(\mathrm{M}^{+}\right)$; Elemental Analysis: C, 56.69; H, 4.79; N, 16.88\%.

\subsection{Synthesis of N-(4-Acetylphenyl)-2-(benzo[d]oxazol-2-ylthio)acetamide (Comp.4)}

$\mathrm{N}$-(4-acetylphenyl)-2-chlroacetamide (2) $(0.01 \mathrm{~mol}, 211.5 \mathrm{gm} / \mathrm{mol}, 2.11 \mathrm{gm})$ was further reacted with 2-mercatobenoxazole (3) $(0.01 \mathrm{~mol}, 151.20 \mathrm{gm} / \mathrm{mol}, 1.51 \mathrm{gm})$. The reaction was stirred at room temperature for $4 \mathrm{hrs}$ in presence of K2CO3 $(0.02 \mathrm{~mol}, 138 \mathrm{gm} / \mathrm{mol}, 2.76 \mathrm{gm})$ and acetone $(25 \mathrm{ml})$ as a reaction medium. The completion of reaction was monitored by TLC using Toluene: Acetone (8:2) as a mobile phase, product was poured into water and stirred for $1 \mathrm{hr}$. The obtained precipitate were collected and dried. The product was crystallized into methanol.

Compound 4: Solid cream yellow; Yield: $81 \%$; M.P.: $164{ }^{\circ} \mathrm{C}$; IR ( $v_{\max }, \mathrm{cm}^{-1}$, ATR): $1413(\mathrm{C}=\mathrm{C}$ str. aromatic ring), $1640(\mathrm{C}=\mathrm{O}$ str.), 2750 (CH2 str. methylene), 3049 (CH str. Aromatic ring), 3232 (NH str.); 1H NMR (400 MHz, DMSO-d6, $\delta$, ppm): 2.56 (3H, s, -CH3), $4.89(2 \mathrm{H}, \mathrm{s},-\mathrm{CH} 2), 7.30-8.10(8 \mathrm{H}, \mathrm{d}, \mathrm{Ar}-\mathrm{H}), 9.21(1 \mathrm{H}, \mathrm{s},-\mathrm{NH}) ; 13 \mathrm{C} \mathrm{NMR}(400 \mathrm{MHz}$, DMSO-d6, d, ppm): 27.2 (C17), 39.2 (C8), 112.6(C2), 121.3(C5), 121.0 (C11), 121.0(C15) , 124.1(C3) , 124.1(C4) , 129.2(C12) , 129.2 (C14), 137.2 (C13), 152.3 (C1), 141.8.0 (C6), 142.2 (C10), 153.8 (C7), 166.0 (C9), 198.2 (C16); MS (m/z): $326\left(\mathrm{M}^{+}\right)$; Elemental Analysis: C, $59.55 ; \mathrm{H}, 4.22 ; \mathrm{N}, 08.35 \%$.

\subsection{General procedure for the synthesis of the title compounds $5 \mathrm{a}-\mathbf{I}$}

N-(4-Acetylphenyl)-2-(benzo[d]oxazol-2-ylthio)acetamide (Comp.4) (0.01 mol, $326 \mathrm{gm} / \mathrm{mol}, 3.26 \mathrm{gm})$ was further treated with different substituted acid hydrazide $(0.01 \mathrm{~mol})$ in ethanol $(20 \mathrm{ml})$ in the presence of catalytic amount of acetic acid and 4-5 drops of fused sodium acetate and refluxed for 8 hours. The completion of the reaction was monitored by the TLC using Toluene : Acetone (8:2) as mobile phase. Resulting solid was separated out, filtered, and washed with water, dried and crystallized by alcohol (99.9\%) [15]. Melting points of each synthesized compound were measured by electrical melting point apparatus. The products were designated as $5 \mathrm{a}-1$ and characterized by elemental, IR, NMR, CMR and MS analyses.

\subsubsection{Physical constant and characterization of 2-(benzo[d]oxazol-2-ylthio)-N-(4-(1-(2- benzoylhydrazono)ethyl)phenyl) acetamide (5a)}

Yield: $72 \%$; M.P.: $194-196{ }^{\circ}$ C; $1 \mathrm{H}$ NMR (400 MHz, DMSO- $\delta 6, \delta$, ppm): $2.32(3 \mathrm{H}$, s, $\left.-\mathrm{CH}_{3}\right), 4.10\left(2 \mathrm{H}, \mathrm{s},-\mathrm{CH}_{2}\right), 7.60-8.09(13 \mathrm{H}, \mathrm{d}, \mathrm{Ar}-\mathrm{H}), 7.16(1 \mathrm{H}, \mathrm{s},-\mathrm{NH}) ; 13 \mathrm{C}$ NMR (400 
MHz, DMSO- $\delta 6, \delta$, ppm): 16.9, 38.4, 110.7, 119.5, 121.7, 121.7, 123.5, 124.5, 127.8, 127.8, $128.6,128.6,129.7,129.7,132.3,132.8,133.4,140.7,141.5,147.8,151.6,163.6,165.1$, 168.2; MS (m/z): $444\left(\mathrm{M}^{+}\right)$; Elemental Analysis: C, 64.23; H, 4.57; N, 12.66\%.

2.3.2. Physical constant and characterization of 2-(benzo[d]oxazol-2-ylthio)-N-(4-(1-(2(3-nitrobenzoyl)hydrazono)ethyl)phenyl)acetamide (5b)

Yield: $74 \%$; M.P.: $205-207{ }^{\circ} \mathrm{C}$; $1 \mathrm{H}$ NMR (400 MHz, DMSO- $66, \delta$, ppm): $2.33(3 \mathrm{H}$, s, $\left.-\mathrm{CH}_{3}\right), 4.09\left(2 \mathrm{H}, \mathrm{s},-\mathrm{CH}_{2}\right), 7.58-8.75(12 \mathrm{H}, \mathrm{d}, \mathrm{Ar}-\mathrm{H}), 7.28(1 \mathrm{H}, \mathrm{s},-\mathrm{NH}) ; 13 \mathrm{C}$ NMR (400 MHz, DMSO- $\delta 6, \delta$, ppm): 16.7, 38.5, 110.6, 119.1, 121.7, 121.7. 123.3, 123.8, 124.8, 127.3, 129.4, 129.4, 129.7, 132.1, 133.1, 133.6, 135.1, 140.8, 141.5, 148.0, 151.9, 163.2, 165.0, 168.2; MS (m/z): $490\left(\mathrm{M}^{+}\right)$; Elemental Analysis : C, 58.93; H, 3.92; N, 14.24\%.

\subsubsection{Physical constant and characterization of 2-(benzo[d]oxazol-2-ylthio)-N-(4-(1-(2-} (4-nitrobenzoyl)hydrazono)ethyl)phenyl) acetamide (5c)

Yield: $77 \%$; M.P.: $210-212{ }^{\circ} \mathrm{C}$; $1 \mathrm{H}$ NMR (400 MHz, DMSO- $\left.\delta 6, \delta, p p m\right): 2.33(3 \mathrm{H}$, s, $\left.-\mathrm{CH}_{3}\right), 4.05\left(2 \mathrm{H}, \mathrm{s},-\mathrm{CH}_{2}\right), 7.60-8.45(12 \mathrm{H}, \mathrm{d}, \mathrm{Ar}-\mathrm{H}), 7.16(1 \mathrm{H}, \mathrm{s},-\mathrm{NH}) ; 13 \mathrm{C}$ NMR $(400$ MHz, DMSO- $\delta 6, \delta$, ppm): 16.9, 39.4, 110.3, 119.7, 121.7, 121.7, 123.7 124.2, 124.2, 125.0, $128.9,128.9,129.2,129.2,133.4,137.6,138.6,141.5,147.5,151.4,151.9,163.4,165.2$, 168.3; MS (m/z): $489\left(\mathrm{M}^{+}\right)$; Elemental Analysis: C, 58.43; H, 3.94; N, 14.52\%.

\subsubsection{Physical constant and characterization of 2-(benzo[d]oxazol-2-ylthio)-N-(4-(1-(2-} (3-methylbenzoyl)hydrazono)ethyl)phenyl) acetamide (5d)

Yield: $65 \%$; M.P.: $204-206{ }^{\circ} \mathrm{C}$; $1 \mathrm{H}$ NMR (400 MHz, DMSO- $\delta 6, \delta$, ppm): 2.30-2.36 $\left(6 \mathrm{H}, \mathrm{s},-\mathrm{CH}_{3}\right), 4.08\left(2 \mathrm{H}, \mathrm{s},-\mathrm{CH}_{2}\right), 7.32-7.95(12 \mathrm{H}, \mathrm{d}, \mathrm{Ar}-\mathrm{H}), 7.34(1 \mathrm{H}, \mathrm{s},-\mathrm{NH}) ; 13 \mathrm{C} \mathrm{NMR}$ (400 MHz, DMSO- $\delta 6, \delta$, ppm): 16.4, 21.8, 38.9, 114.8, 116.2, 121.4, 121.4, 123.3, 123.8, $124.5,128.1,128.4,129.6,129.6,131.5,134.5,136.6,137.9,138.2,139.3,140.0,145.9$, 147.4, 163.6, 167.6; MS (m/z): $458\left(\mathrm{M}^{+}\right)$; Elemental Analysis: C, 65.66; H, 4.74; N, 12.30\%.

2.3.5. Physical constant and characterization of 2-(benzo[d]oxazol-2-ylthio)-N-(4-(1-(2(4-methylbenzoyl)hydrazono)ethyl)phenyl) acetamide (5e)

Yield: $66 \%$; M.P.: $202-204{ }^{\circ} \mathrm{C}$; $1 \mathrm{H}$ NMR (400 MHz, DMSO- $\delta 6, \delta$, ppm): 2.30-2.37 $\left(6 \mathrm{H}, \mathrm{s},-\mathrm{CH}_{3}\right), 4.14\left(2 \mathrm{H}, \mathrm{s},-\mathrm{CH}_{2}\right), 7.36-7.98(12 \mathrm{H}, \mathrm{d}, \mathrm{Ar}-\mathrm{H}), 6.91(1 \mathrm{H}, \mathrm{s},-\mathrm{NH}) ; 13 \mathrm{C} \mathrm{NMR}$ (400 MHz, DMSO- $\delta 6, \delta$, ppm): 16.8, 21.2, 38.4, 110.5, 119.2, 121.4, 121.4, 123.7, 124.6, $127.3,127.3,129.1,129.1,129.5,129.5,129.9,133.4,140.7,141.6,141.8,147.6,151.9$, 163.3, 165.2, 168.4; MS (m/z): $459\left(\mathrm{M}^{+}\right)$; Elemental Analysis: C, 65.45; H, 4.82; N, 12.35\%.

2.3.6. Physical constant and characterization of 2-(benzo[d]oxazol-2-ylthio)-N-(4-(1-(2(4-methoxybenzoyl)hydrazono)ethyl)phenyl) acetamide (5f)

Yield: $66 \%$; M.P.: $210-212{ }^{\circ} \mathrm{C}$; $1 \mathrm{H}$ NMR (400 MHz, DMSO- $\delta 6, \delta$, ppm): 2.29-3.85 $\left(6 \mathrm{H}, \mathrm{s},-\mathrm{CH}_{3}\right), 4.08\left(2 \mathrm{H}, \mathrm{s},-\mathrm{CH}_{2}\right), 7.15-7.98(12 \mathrm{H}, \mathrm{d}, \mathrm{Ar}-\mathrm{H}), 7.22(1 \mathrm{H}, \mathrm{s},-\mathrm{NH}) ; 13 \mathrm{C}$ NMR (400 MHz, DMSO- $\delta 6, \delta$, ppm): 16.8, 38.4, 55.3, 110.5, 114.5, 114.5, 119.2, 121.4, 121.4, 
$123.7,124.6,128.3,128.3,129.5,129.5,129.9,133.4,140.7,141.6,147.6,151.9,163.3$, 164.1, 165.2, 168.4; MS (m/z): $474\left(\mathrm{M}^{+}\right)$; Elemental Analysis: C, 63.37; H, 4.82; N, 11.85\%.

2.3.7. Physical constant and characterization of 2-(benzo[d]oxazol-2-ylthio)-N-(4-(1-(2(2-chlorobenzoyl)hydrazono)ethyl)phenyl) acetamide (5g)

Yield: $70 \%$; M.P.: $212-214{ }^{\circ} \mathrm{C}$; $1 \mathrm{H}$ NMR (400 MHz, DMSO- $\delta 6, \delta$, ppm): $2.36(3 \mathrm{H}$, s, $\left.-\mathrm{CH}_{3}\right), 4.14\left(2 \mathrm{H}, \mathrm{s},-\mathrm{CH}_{2}\right), 7.41-8.04(12 \mathrm{H}, \mathrm{d}, \mathrm{Ar}-\mathrm{H}), 7.14(1 \mathrm{H}, \mathrm{s},-\mathrm{NH}) ; 13 \mathrm{C}$ NMR (400 MHz, DMSO- $\delta 6, \delta$, ppm): 16.8, 38.4, 110.5, 119.2, 121.4, 121.4, 124.6, 126.3, 128.6, 129.5 , $129.5,129.9,130.2,132.3,133.3,133.6,134.7,140.7,147.6,151.9,163.3,164.1,165.2$, 168.4 ; MS (m/z): $480\left(\mathrm{M}^{+}\right)$; Elemental Analysis: C, 61.02; H, 4.04; N, 11.72\%.

2.3.8. Physical constant and characterization of 2-(benzo $[d]$ oxazol-2-ylthio)-N-(4-(1-(2(3-chlorobenzoyl)hydrazono)ethyl)phenyl) acetamide (5h)

Yield: $71 \%$; M.P.: $214-216{ }^{\circ} \mathrm{C}$; $1 \mathrm{H}$ NMR (400 MHz, DMSO- $\delta 6, \delta$, ppm): $2.32(3 \mathrm{H}$, s, $\left.-\mathrm{CH}_{3}\right), 4.08\left(2 \mathrm{H}, \mathrm{s},-\mathrm{CH}_{2}\right), 7.40-7.99(12 \mathrm{H}, \mathrm{d}, \mathrm{Ar}-\mathrm{H}), 7.05(\mathrm{H}, \mathrm{s},-\mathrm{NH}) ; 13 \mathrm{C}$ NMR (400 MHz, DMSO- $\delta 6, \delta$, ppm): 16.4, 39.4, 110.6, 119.4, 121.6, 121.6, 124.3, 126.3, 128.9, 129.9, $129.9,130.1,130.6,132.6,133.5,133.5,134.8,140.4,147.6,151.6,163.8,164.2,165.5$, 168.0; MS (m/z): $480\left(\mathrm{M}^{+}\right)$; Elemental Analysis: C, 60.21; H, 4.14; N, 11.73\%.

2.3.9. Physical constant and characterization of 2-(benzo[d]oxazol-2-ylthio)-N-(4-(1-(2(4-chlorobenzoyl)hydrazono)ethyl)phenyl) acetamide (5i)

Yield: $71 \%$; M.P.: $210-212{ }^{\circ} \mathrm{C}$; $1 \mathrm{H}$ NMR (400 MHz, DMSO- $\delta 6, \delta$, ppm): $2.32(3 \mathrm{H}$, s, $\left.-\mathrm{CH}_{3}\right), 4.05\left(2 \mathrm{H}, \mathrm{s},-\mathrm{CH}_{2}\right), 7.40-8.04(12 \mathrm{H}, \mathrm{d}, \mathrm{Ar}-\mathrm{H}), 7.27(1 \mathrm{H}, \mathrm{s},-\mathrm{NH}) ; 13 \mathrm{C}$ NMR (400 MHz, DMSO- $\delta 6, \delta$, ppm): 16.6, 39.6, 110.8, 119.4, 121.6, 121.6, 124.4, 128.9, 128.9, 129.9, $129.9,130.1,130.1,132.7,133.7,133.7,134.9,140.4,147.6,151.9,163.7,164.2,165.5$, 168.2; MS (m/z): $479\left(\mathrm{M}^{+}\right)$; Elemental Analysis: C, 60.23; H, 4.13; N, 11.62\%.

2.3.10. Physical constant and characterization of 2-(benzo[d]oxazol-2-ylthio)-N-(4-(1-(2(2-hydroxybenzoyl)hydrazono)ethyl)phenyl) acetamide (5j)

Yield: $65 \%$; M.P.: $216-218{ }^{\circ} \mathrm{C}$; $1 \mathrm{H}$ NMR (400 MHz, DMSO- $\delta 6, \delta$, ppm): $2.32(3 \mathrm{H}$, $\left.\mathrm{s},-\mathrm{CH}_{3}\right), 5.34(1 \mathrm{H}, \mathrm{s},-\mathrm{OH}), 4.06\left(2 \mathrm{H}, \mathrm{s},-\mathrm{CH}_{2}\right), 7.12-7.78(12 \mathrm{H}, \mathrm{d}, \mathrm{Ar}-\mathrm{H}), 7.19(1 \mathrm{H}, \mathrm{s},-\mathrm{NH})$; 13C NMR (400 MHz, DMSO- $\delta 6, \delta, \mathrm{ppm}): 16.2,38.5,110.3,117.4,117.9,119.4,121.2$, $121.7,121.7,123.5,124.6,128.9,129.3,133.1,133.6,140.9,141.7,147.8,151.6,153.4$, 159.8, 163.5, 165.0, 165.4; MS (m/z): $460\left(\mathrm{M}^{+}\right)$; Elemental Analysis: $\mathrm{C}, 62.45 ; \mathrm{H}, 4.38$; N, $12.19 \%$.

2.3.11. Physical constant and characterization of 2-(benzo $[d]$ oxazol-2-ylthio)-N-(4-(1-(2(3-hydroxybenzoyl)hydrazono)ethyl)phenyl) acetamide (5k)

Yield: $61 \%$; M.P.: $226-228^{\circ} \mathrm{C}$; $1 \mathrm{H}$ NMR (400 MHz, DMSO- $\delta 6, \delta$, ppm): $2.32(3 \mathrm{H}$, $\left.\mathrm{s},-\mathrm{CH}_{3}\right), 5.32(1 \mathrm{H}, \mathrm{s},-\mathrm{OH}), 4.15\left(2 \mathrm{H}, \mathrm{s},-\mathrm{CH}_{2}\right), 7.10-7.82(12 \mathrm{H}, \mathrm{d}, \mathrm{Ar}-\mathrm{H}), 7.34(1 \mathrm{H}, \mathrm{s},-\mathrm{NH})$; 13C NMR (400 MHz, DMSO- $\delta 6, \delta, \mathrm{ppm}): 16.6,38.7,110.3,117.6,117.6,119.4,121.1$, 
$121.8,121.8,123.3,124.4,128.7,129.3,133.0,133.7,140.8,141.7,147.9,151.5,153.4$, 159.6, 163.8, 165.0, 165.5; MS (m/z): $461\left(\mathrm{M}^{+}\right)$; Elemental Analysis: $\mathrm{C}, 62.24 ; \mathrm{H}, 4.26$; N, $12.22 \%$.

\subsubsection{Physical constant and characterization of 2-(benzo[d]oxazol-2-ylthio)-N-(4-(1-(2- (4-hydroxybenzoyl)hydrazono)ethyl)phenyl) acetamide (5l)}

Yield: $67 \%$; M.P.: $220-222{ }^{\circ} \mathrm{C}$; $1 \mathrm{H}$ NMR (400 MHz, DMSO- $\delta 6, \delta$, ppm): $2.25(3 \mathrm{H}$, $\left.\mathrm{s},-\mathrm{CH}_{3}\right), 5.37(1 \mathrm{H}, \mathrm{s},-\mathrm{OH}), 4.12\left(2 \mathrm{H}, \mathrm{s},-\mathrm{CH}_{2}\right), 7.04-7.77(12 \mathrm{H}, \mathrm{d}, \mathrm{Ar}-\mathrm{H}), 7.04(1 \mathrm{H}, \mathrm{s},-\mathrm{NH})$; 13C NMR (400 MHz, DMSO- $\delta 6, \delta, \mathrm{ppm}): 16.4,38.6,110.4,116.2,116.2,119.4,121.8$, $121.8,123.9,124.6,125.5,128.9,128.9,129.5,129.5,133.2,140.9,141.4,147.8,151.9$, 161.9, 163.5, 165.2, 168.3; MS (m/z): $460\left(\mathrm{M}^{+}\right)$; Elemental Analysis: C, 62.62; H, 4.56; N, $12.46 \%$.

\section{RESULTS AND DISCUSSION}

Scheme 1 was completed by three different steps and final compounds were designated at 5a-1. Compounds $5 \mathrm{a}-1$ has not been reported previously confirmed by using the Scifinder search. The structures of all compounds were confirmed by the spectroscopy like FT-IR, $1 \mathrm{H}$ NMR, 13C NMR, MS and CHN analyzer. The data of FT-IR spectroscopy provides valuable information regarding the nature of functional group attached. In order to study the bonding mode of compound 4 to the compound $5 \mathrm{a}-1$, the IR spectrum of compound 4 was compared with the spectra of compound $5 \mathrm{a}-1$. Considerable differences to be expected were observed. The general structure of compounds 5a-1 designated as below, where $\mathrm{R}$ is different substitute group or atom.

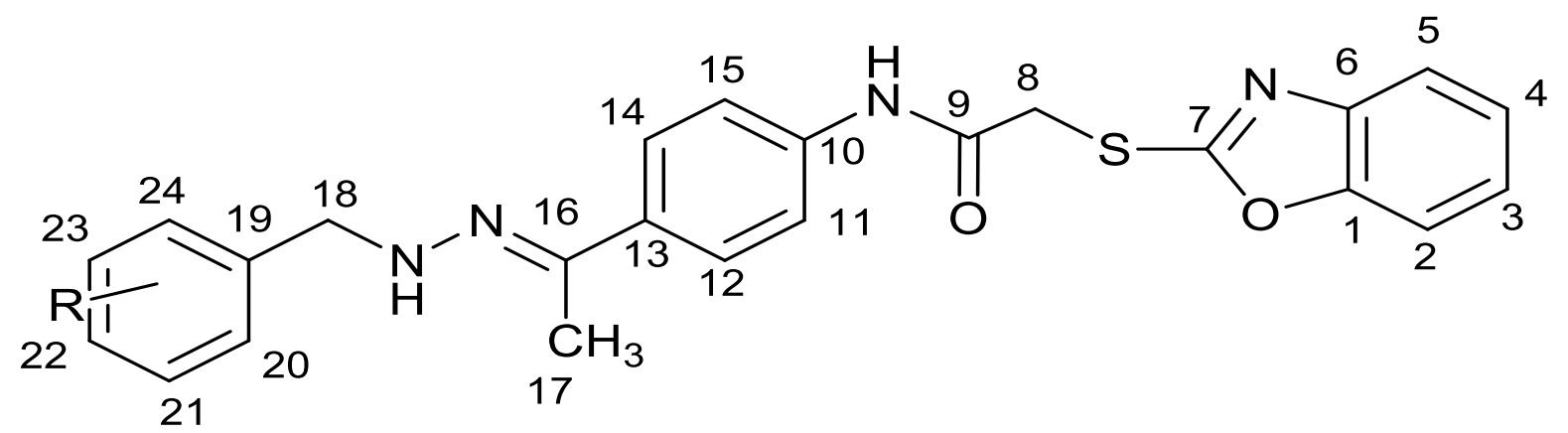

Figure 1. Compound 5a-1.

The structures of the final compounds $5 \mathrm{a}-1$ were established by their spectral analysis. Using compound 5a as a representative example, its FTIR spectrum of 5a showed the most relevant peaks of benzooxazole-acetylphenyl ring. Stretching vibration at $3223 \mathrm{~cm}^{-1}$ indicates that compound containing a secondary amine. The vibration at $1584 \mathrm{~cm}^{-1}$ and $3052 \mathrm{~cm}^{-1}$ over the range showed intensity absorption peaks corresponding to aromatic $\mathrm{C}-\mathrm{H}$ stretching vibrations. The absorption peaks at $1658 \mathrm{~cm}^{-1}$ is due to the carbonyl groups present structure as amide group.

$1 \mathrm{H}$ NMR It has been observed from the chemical structure of compound that C-11,C$12, \mathrm{C}-14$ and C-15 are pairs of chemically equivalent protons which appear at $\delta=7.69 \mathrm{ppm}$ 
and $\delta=7.85 \mathrm{ppm}$ indicates that this all four carbon are presence in same aromatic ring. As well as C-2, C-3, C-4 and C-5 are pairs of chemically equivalent protons which appeared at $\delta$ $=7.66 \mathrm{ppm}$ and $\delta=7.77 \mathrm{ppm}$ value indicates that benzooxazole ring. C-20, C-21, C-22, C-23 and $\mathrm{C}-24$ are also pairs of chemically equivalent protons which indicates that this all carbon presence of same aromatic ring.

The protons attached at C-8 appeared as a singlet at $\delta=4.02 \mathrm{ppm}$ due to sulfur and C-9 carbonyl group atmosphere. Carbon C-17 contains a proton gives singlet at $\delta=2.35 \mathrm{ppm}$ indicates that azomethine group present in the structure. The mass spectrum of 5 a showed a molecular ion peak at $\mathrm{m} / \mathrm{z} 444(\mathrm{M}+1)$ which is in agreement with its proposed structure.

Table 1. Antimicrobial screening results of compounds 2,4 and $\mathbf{5 a}-\mathbf{k}$.

\begin{tabular}{|c|c|c|c|c|c|c|c|}
\hline \multirow[t]{3}{*}{ Entry } & \multirow[t]{3}{*}{$\mathbf{R}$} & \multicolumn{6}{|c|}{ Minimum inhibitory concentration (MIC) $\mu \mathrm{g} / \mathrm{ml}$} \\
\hline & & \multicolumn{2}{|c|}{$\frac{\text { Gram positive }}{\underline{\text { bacteria }}}$} & \multicolumn{2}{|c|}{$\frac{\text { Gram negative }}{\underline{\text { bacteria }}}$} & \multicolumn{2}{|c|}{ Fungi } \\
\hline & & S. aureus & S. pyogenes & E. coli & $\begin{array}{c}\mathrm{P} . \\
\text { aeruginosa }\end{array}$ & $\begin{array}{c}\text { C. } \\
\text { albicans }\end{array}$ & A. niger \\
\hline 2 & - & $>1000$ & $>1000$ & $>1000$ & $>1000$ & $>1000$ & $>1000$ \\
\hline 4 & - & 1000 & 1000 & 1000 & 1000 & 1000 & 1000 \\
\hline $5 \mathbf{a}$ & $-\mathrm{H}$ & 250 & 250 & 500 & 500 & 200 & 100 \\
\hline $\mathbf{5 b}$ & $3-\mathrm{NO}_{2}$ & 50 & 50 & 50 & 100 & 100 & 500 \\
\hline $5 c$ & $4-\mathrm{NO}_{2}$ & 25 & 25 & 25 & 25 & 50 & 100 \\
\hline $5 d$ & $3-\mathrm{CH}_{3}$ & 500 & 500 & 500 & 500 & 500 & 500 \\
\hline $5 e$ & $4-\mathrm{CH}_{3}$ & 500 & 500 & 500 & 250 & 500 & 500 \\
\hline $5 f$ & $4-\mathrm{OCH}_{3}$ & 500 & 500 & 500 & 500 & 1000 & 500 \\
\hline $5 g$ & $2-\mathrm{Cl}$ & 25 & 100 & 50 & 100 & 25 & 50 \\
\hline $5 \mathrm{~h}$ & $3-\mathrm{Cl}$ & 100 & 100 & 100 & 250 & 50 & 100 \\
\hline $5 \mathbf{i}$ & $4-\mathrm{Cl}$ & 200 & 200 & 200 & 250 & 200 & 200 \\
\hline $5 \mathbf{j}$ & $2-\mathrm{OH}$ & 500 & 500 & 500 & 500 & 500 & 500 \\
\hline $5 k$ & $3-\mathrm{OH}$ & 200 & 500 & 500 & 500 & 500 & 1000 \\
\hline 51 & $4-\mathrm{OH}$ & 200 & 500 & 250 & 200 & 500 & 1000 \\
\hline $\begin{array}{l}\text { Chlora- } \\
\text { mphenicol }\end{array}$ & & 50 & 50 & 50 & 50 & - & - \\
\hline $\begin{array}{c}\text { Ketoco- } \\
\text { nazole }\end{array}$ & & - & - & - & - & 50 & 50 \\
\hline
\end{tabular}

Minimum Inhibitory Concentration for bacteria $(\mathrm{MICb})$ of all the newly synthesized compounds was determined for their in vitro antimicrobial activity against different bacterial and fungal strains by the conventional broth-dilution method [16] using standard drugs which aer chloramphenicol and ketoconazole. The results of antimicrobial studies are presented in Table 1. Intermediates 2 and 4 showed poor antimicrobial activity against all tested bacterial and fungal strains as compared to final derivatives $5 \mathrm{a}-1$. Newly synthesized derivatives showed improved antibacterial activity compared to antifungal activity. Compounds $5 \mathrm{~b}, 5 \mathrm{c}$ 
and $5 \mathrm{~g}$ were found to be highly active against all the bacterial strains, showing inhibition in the range of $25-100 \mathrm{mg} / \mathrm{ml}$.

Among them, compounds $5 \mathrm{c}$ emerged as the most effective antibacterial agents with a 2 to 4-fold higher MIC $(25 \mathrm{mg} / \mathrm{ml})$ than the reference drug Chloramphenicol. Compounds $5 \mathrm{~b}$, $5 \mathrm{c}$ and $5 \mathrm{~g}$ exhibited comparable antibacterial activity with MIC values of $25-100 \mathrm{mg} / \mathrm{ml}$. Compounds $5 \mathrm{c}$ and $5 \mathrm{i}$ substituted with inductively electron withdrawing nitro and chloro groups respectively, at the para position showed the highest antibacterial activity $(\mathrm{NO} 2>\mathrm{Cl})$. The presence of electron donating groups on the phenyl ring resulted in a significant decrease in antimicrobial activity of compounds $5 \mathrm{~d}, 5 \mathrm{e}, 5 \mathrm{f}, 5 \mathrm{j}, 5 \mathrm{k}$ and 51.

From these results, it can be observed that the antibacterial activity was considerably affected by the substitution pattern on the phenyl ring. Further, the results of the antifungal activity indicated that compound $5 \mathrm{~g}$ endowed with chlorine emerged as the most effective antifungal agent and showed an MIC in the range of $25-50 \mathrm{mg} / \mathrm{ml}$ against three fungal strains using ketoconazole as a positive control.

\section{CONCLUSION}

From the study of above results from all the spectral and physical data of the synthesized compounds clearly conclude that designed compounds were synthesized successfully. We have accomplished the synthesis of new derivatives of benzoxazole $5 \mathrm{a}-\mathrm{k}$ having schiff base with the hope of generating new bioactive molecules that could be useful as potent antimicrobial agents.

A series of compounds when substituted by electron-withdrawing group like $\mathrm{NO}_{2}$ and $\mathrm{Cl}$ enhances the antimicrobial activity when present on aromatic ring. On the other side the used of electron donating group didn't enhances the antimicrobial activity when present on aromatic ring. Among the twelve newer derivatives, analogs $5 \mathrm{~b}, 5 \mathrm{c}, 5 \mathrm{~g}, 5 \mathrm{~h}$ and $5 \mathrm{i}$ possessing electron withdrawing atom/group such as Nitro and Chloro at the para or mata position were identified as the most potent antibacterial agents and compound $5 \mathrm{~g}$ was found to be the most effective antifungal agent. The results lead us to further studies to acquire more information about newer derivatives of benzoxazole.

\section{Acknowledgments}

We would like to express our sincere gratitude to The Sheth M. N. Science College, H.N.G.U., Patan for providing us laboratory facilities. The authors are thankful Saurastra University, Rajkot and CMCRI, Bhavnagar for providing analytical data of the compounds. Authors are also thankful to Dr. Deepkumar S. Joshi for his enlightenment.

\section{References}

[1] T. Panneer Selvam, P. P. Radhika, S. Janagaraj, A. Siva Kumar, Research in biotechnology 2 (2011) 50-57.

[2] Pedrazzini S, De Angelis M, Muciaccia WZ, Sacchi C, Forgione A, ArzneimittelForschung 38 (8): 1170-5. PMID 3196413

[3] Samia M. Rida, Fawzia A. Ashour, Soad A.M. El-Hawash, Mona M. ElSemary, Mona H. Badr, Manal A. Shalaby, Eur J Med Chem 40 (2005) 949-959. 
[4] Jarmila Vinsova, Vaclav Horak, Vladimir Buchta and Jarmila Kaustova, Molecules 10 (2005) 783-793.

[5] Meric Koksal, Nesrin Gokhan, Esra Kupeli, Erdem Yesilada, and Hakki Erdogan, Arch Pharm Res. 30 (2007) 419-424.

[6] Mimnaugh, E.G.; Xu, W.; Vos, M. Mol. Cancer Ther. 3 (2004) 551-566.

[7] Yadav, R; Sirvastava, SD and Sirvastava, SK (2005), Indian J. Chem. 44 (2005) 12621266.

[8] Ramón, G; Domenech, B; Ana, C; Gregori, C and Calabuig, C, Internet Electron. J. Mol. Des., BioChem Press, 1 (2002) 339-350.

[9] R. V. Satyendra, K. A. Vishnumurthy, H. M. Vagdevi, K. P. Rajesh, H. Manjunatha, and A. Shruthi, European Journal of Medicinal Chemistry 46 (2011) 3078-3084.

[10] Paramashivappa R, Phanikumar P, Subbarao P, Srinivasarao A, Bioorg Med Chem Lett. 13, 2003, 657-660.

[11] Grocer, H., Kus, C., Boykin, D.W., Yildiz, S., Altanlar, N., Bioorg. Med. Chem. 10 (2002) 2589-2596.

[12] Nicholson RM, Murphy JR, Dearden JR, Journal of Pharmacy and Pharmacology 34 (1982) 106-111.

[13] Fang, B., Zhou, C.H., Rao, X.C., Eur. J. Med. Chem. 45 (2010) 4388-4398.

[14] E. F. Magomedova, V. V. Pinyaskin, A. Sh. Aminova, Pharma. Chem. J. 41 (2007) 474-475.

[15] Kalpesh Parikh, Deepkumar Joshi, Med Chem Res. 22 (2013) 3688-3697.

[16] P.C. Hannan, Vet. Res. 31 (2000) 373-395. 